\title{
Dok-7 regulates neuromuscular synapse formation by recruiting Crk and Crk-L
}

\author{
Peter T. Hallock, ${ }^{1}$ Chong-Feng Xu, ${ }^{2}$ Tae-Ju Park, ${ }^{3}$ Thomas A. Neubert, ${ }^{2}$ Tom Curran, ${ }^{3}$ \\ and Steven J. Burden ${ }^{1,4}$

\begin{abstract}
${ }^{1}$ Molecular Neurobiology Program, Skirball Institute of Biomolecular Medicine, New York University Medical School, New York, New York 10016, USA; ${ }^{2}$ Structural Biology Program, Skirball Institute of Biomolecular Medicine, New York University Medical School, New York, New York 10016, USA; ${ }^{3}$ Department of Pathology and Laboratory Medicine, The Children's Hospital of Philadelphia, Boulevard, Philadelphia, Pennsylvania 19104, USA
\end{abstract}

\begin{abstract}
Agrin, released by motor neurons, promotes neuromuscular synapse formation by stimulating MuSK, a receptor tyrosine kinase expressed in skeletal muscle. Phosphorylated MuSK recruits docking protein-7 (Dok-7), an adaptor protein that is expressed selectively in muscle. In the absence of Dok-7, neuromuscular synapses fail to form, and mutations that impair Dok-7 are a major cause of congenital myasthenia in humans. How Dok-7 stimulates synaptic differentiation is poorly understood. Once recruited to MuSK, Dok-7 directly stimulates MuSK kinase activity. This unusual activity of an adapter protein is mediated by the $\mathrm{N}$-terminal region of Dok-7, whereas most mutations that cause congenital myasthenia truncate the C-terminal domain. Here, we demonstrate that Dok-7 also functions downstream from MuSK, and we identify the proteins that are recruited to the C-terminal domain of Dok-7. We show that Agrin stimulates phosphorylation of two tyrosine residues in the C-terminal domain of Dok-7, which leads to recruitment of two adapter proteins: Crk and Crk-L. Furthermore, we show that selective inactivation of $C r k$ and $C r k-L$ in skeletal muscle leads to severe defects in neuromuscular synapses in vivo, revealing a critical role for Crk and Crk-L downstream from Dok-7 in presynaptic and postsynaptic differentiation.
\end{abstract}

[Keywords: Neuromuscular synapse; acetylcholine receptor; congenital myasthenia; receptor tyrosine kinase; synapse formation; mouse development]

Supplemental material is available at http://www.genesdev.org.

Received August 3, 2010; revised version accepted September 10, 2010.

Neuromuscular synapse formation requires a complex exchange of signals between motor neurons and skeletal myofibers (Sanes and Lichtman 2001). Agrin, supplied by motor neurons, binds to the low-density lipoprotein receptor (LDLR)-related protein 4 (Lrp4), a member of the LDLR family (Kim et al. 2008; Zhang et al. 2008). Lrp4 selfassociates, forms a complex with MuSK, and is essential for neuromuscular synapse formation (Weatherbee et al. 2006). Binding between Agrin and Lrp4 stimulates tyrosine phosphorylation of MuSK, a receptor tyrosine kinase expressed in skeletal muscle (Jennings et al. 1993; Valenzuela et al. 1995; Kim et al. 2008; Zhang et al. 2008). Once phosphorylated, MuSK recruits docking protein-7 (Dok-7), a recently discovered noncatalytic adapter protein containing N-terminal pleckstrin homology $(\mathrm{PH})$ and phosphotyrosinebinding (PTB) domains, required to bind tyrosine phosphorylated MuSK, as well as a C-terminal domain (Okada et al. 2006). In the absence of Dok-7, like in the absence of MuSK, neuromuscular synapses fail to form /Okada et al.

${ }^{4}$ Corresponding author.

E-MAIL burden@saturn.med.nyu.edu; FAX (212) 263-8214.

Article is online at http://www.genesdev.org/cgi/doi/10.1101/gad.1977710.
2006; Vogt et al. 2009). Furthermore, hypomorphic mutations in human Dok-7 that impair Dok-7 function cause congenital myasthenia (CMS), characterized by structurally and functionally defective synapses, leading to muscle weakness and fatigue (Beeson et al. 2006; Muller et al. 2007; Selcen et al. 2008). Although mutations in Agrin or MuSK are uncommon causes of neuromuscular disease, mutations in Dok-7 represent a major form of CMS. As such, understanding how Dok-7 regulates neuromuscular synapse formation is critical not only for understanding the mechanisms of synapse formation, but also for understanding how mutations in Dok-7 lead to disease.

Once recruited to MuSK, Dok-7 stimulates further MuSK phosphorylation and increases MuSK kinase activity (Okada et al. 2006; Inoue et al. 2009; Bergamin et al. 2010). One idea suggests that Dok-7 regulates synapse formation strictly by regulating MuSK phosphorylation, and as such acts as an inside-out ligand for MuSK. Consistent with this idea, the $\mathrm{PH} / \mathrm{PTB}$ domains of Dok-7 form a dimer that binds MuSK, promotes the formation of MuSK dimers, and stimulates MuSK phosphorylation in vitro (Bergamin et al. 2010). Moreover, certain disease-causing mutations in the PTB domain of human Dok-7 impair 
Dok-7 dimerization, demonstrating that Dok-7 dimerization is critical to activate MuSK in vivo (Bergamin et al. 2010). Nonetheless, a majority of patients with Dok-7 CMS carry mutations that truncate the C-terminal domain, suggesting that the C-terminal domain has an important function separate from the role of the $\mathrm{N}$-terminal domain in regulating MuSK kinase activity. These findings raise the possibility that Dok-7 may engage downstream signaling pathways and transduce MuSK activation to synaptic differentiation.

Here, we identify two tyrosine residues in Dok-7 that are phosphorylated by Agrin stimulation, and show that two proteins, Crk and Crk-L, are recruited to these phosphorylation sites in Dok-7. Moreover, we demonstrate that inactivating Crk and Crk-L in vivo in muscle leads to defects in neuromuscular synapses that are virtually identical to those found in humans expressing truncated forms of Dok-7, which cannot be phosphorylated at these two sites. These findings demonstrate that Dok-7 functions downstream from MuSK, and identify the signaling molecules engaged by Dok-7 to promote synaptic differentiation.

\section{Results}

Agrin stimulates phosphorylation of Dok-7 at Y396 and Y406

Agrin stimulates tyrosine phosphorylation of MuSK, leading to the recruitment of Dok-7 (Okada et al. 2006). To determine whether Agrin stimulates Dok-7 tyrosine phosphorylation, we treated cultured myotubes with Agrin and immunoprecipitated Dok-7, and identified phosphorylated residues by mass spectrometry (MS). Figure 1 shows that Agrin stimulated phosphorylation of Y406 (Fig. 1A; Supplemental Fig. S1A-C). We did not detect phosphorylation of Y396, although Y396 appears to be a good substrate for phosphorylation and is embedded in a sequence resembling that surrounding Y406. As such, we were
A

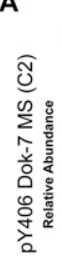
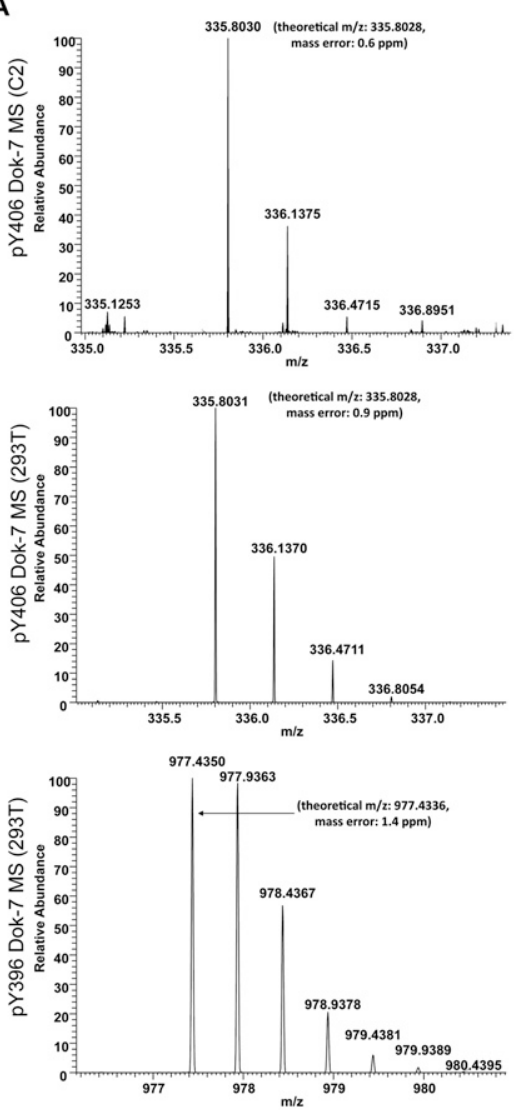

B

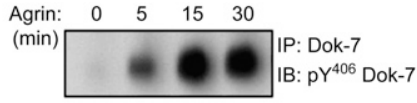

C
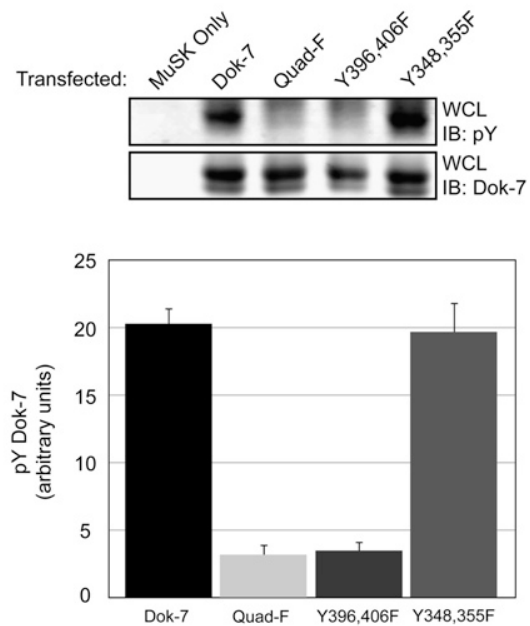

D

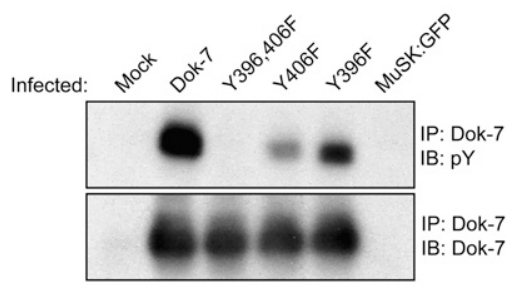

Figure 1. Agrin stimulates phosphorylation of Dok-7 Y396 and Y406. (A) Dok-7 was immunoprecipitated from lysates of C2 myotubes, which had been stimulated with Agrin for $30 \mathrm{~min}$, or from 293 cells transfected with Dok-7 and MuSK. Following SDS-PAGE, Dok-7 was digested with trypsin, and the tryptic fragments were analyzed by LTQ-Orbitrap MS. Agrin stimulated phosphorylation of Y406 in C2 myotubes; both Y396 and Y406 were phosphorylated in 293 cells transfected with Dok-7 and MuSK. (B) Agrin stimulated phosphorylation of Y396 and Y406 in C2 myotubes. $(C)$ Western blots of lysates (WCL) from transfected 293 cells show that Dok-7 was not detectably phosphorylated at Y348 or Y355, indicating that Y396 and Y406 are the only tyrosine phosphorylation sites in reconstituted 293 cells $\mid n=3$; mean \pm SEM). (D) Y396 and Y406 are the major tyrosine phosphorylation sites in Dok-7 expressed in $\mathrm{C} 2$ myotubes. Mutation of either Y396 or Y406 reduced the extent of Agrin-independent Dok-7 phosphorylation. Mutation of both residues reduced phosphorylation to background levels, indicating that Y396 and Y406s are the major, if not only, tyrosine phosphorylation sites. See also Supplemental Figure S1. 
concerned that a failure to detect Y396 phosphorylation might be caused by poor recovery and representation of this peptide by MS. Thus, we used an additional approach to determine whether Y396 is phosphorylated following Agrin stimulation. We stimulated myotubes with Agrin and immunoprecipitated Dok-7, and probed Western blots with antibodies specific for pY396 or pY406 (Fig. 1B; Supplemental Fig. S1D). Figure 1B shows that Agrin stimulated phosphorylation of Y396 and Y406.

To determine whether additional tyrosines are phosphorylated in Dok-7, we analyzed Dok-7 phosphorylation in 293 cells expressing both MuSK and Dok-7. Coexpression of MuSK and Dok-7 in 293 cells activates MuSK and leads to high levels of Dok-7 tyrosine phosphorylation (Okada et al. 2006), providing a sensitive assay to identify Dok-7 phosphorylation sites (Fig. 1C). We expressed MuSK together with wild-type or mutant forms of Dok7-including Dok-7 Y396F/Y406F, Dok-7 Y348F/Y355F, and Dok-7 Y396F/Y406F/Y348F/Y355F-and measured Dok-7 phosphorylation by probing Western blots with antibodies to phosphotyrosine. Figure 1 shows that mutation of Y396 and Y406 reduced Dok-7 phosphorylation to background levels (Fig. 1C). In contrast, mutation of Y348 and Y355 had no effect on Dok-7 phosphorylation (Fig. 1C). Moreover, the level of Dok-7 phosphorylation was similar in cells transfected with a Dok-7 Y396F/ Y406F double mutant and a Dok-7 Y396F/Y406F/Y348F/ Y355F quadruple mutant (Fig. 1C), indicating that Y348 and Y355 were not phosphorylated. Mapping of Dok-7 phosphorylation sites by MS confirmed that Y396 and Y406 were the only tyrosines phosphorylated in 293 cells expressing MuSK and Dok-7 (Fig. 1A). In addition, we expressed these same Dok-7 constructs in C2 myotubes and found that Y396 and Y406 were the major, if not only, tyrosine phosphorylation sites in Dok-7 in muscle (Fig. 1D).

\section{Dok-7 tyrosine phosphorylation is dispensable for MuSK and acetylcholine receptor $\beta$ (AChR- $\beta$ ) subunit tyrosine phosphorylation}

Agrin stimulates tyrosine phosphorylation of the AChR$\beta$ subunit, which is important, although not essential, for clustering AChRs and regulating synaptic differentiation (Friese et al. 2007). Increasing Dok-7 expression in muscle leads to a substantial increase in MuSK phosphorylation, AChR phosphorylation, and AChR clustering (Okada et al. 2006; Hamuro et al. 2008). These and additional experiments indicate that Dok-7 function can be analyzed by introducing wild-type or mutant forms of Dok-7 into wildtype myotubes.

Dok-7 is an unusual adapter protein, since, once recruited to MuSK, Dok-7 stimulates MuSK tyrosine phosphorylation. To determine whether this stimulatory activity of Dok-7 requires phosphorylation of Dok-7 at Y396 or Y406, we transferred wild-type or mutant forms of Dok-7 into cultured myotubes and measured MuSK tyrosine phosphorylation. We found that Dok-7 phosphorylation played only a minor role in regulating the extent of MuSK and AChR phosphorylation, since mutation of Y396 and Y406 reduced MuSK and AChR tyrosine phosphorylation by only $\sim 30 \%$ (Fig. $2 \mathrm{~A}-\mathrm{C}$ ). These data indicate that Dok-7 stimulates MuSK phosphorylation by at least two mechanisms: one that strongly activates MuSK and does not require Dok-7 phosphorylation, and
A

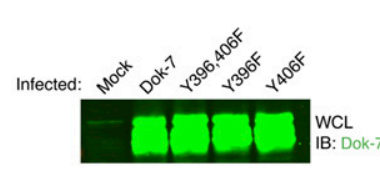

B
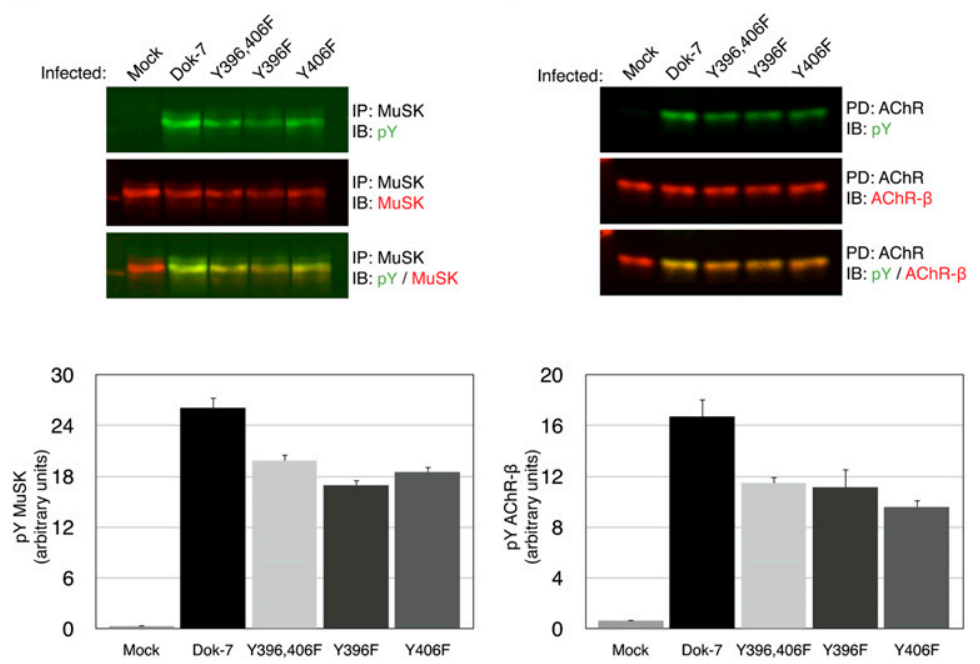

Figure 2. Dok-7 tyrosine phosphorylation is dispensable for MuSK and AChR- $\beta$ subunit tyrosine phosphorylation. (A) Dok-7 was overexpressed $\sim 100$-fold in each C2 muscle cell line $(n=3$; mean \pm SEM). (B) MuSK phosphorylation levels were elevated $\sim 18$-fold in each of the Dok-7 tyrosine mutant lines, compared with mock-infected C2 cells. MuSK phosphorylation levels were modestly higher $(\sim 30 \%)$ in the cell line infected with wild-type Dok-7 than in cell lines expressing any of the Dok-7 tyrosine mutations $(n=3$; mean \pm SEM), indicating that Dok-7 stimulates MuSK activation largely through a mechanism that is independent of Dok-7 phosphorylation. (C) Like MuSK phosphorylation, AChR phosphorylation levels were induced to the same extent in cell lines expressing single or double mutations in Dok-7 and at levels that were modestly reduced $(\sim 30 \%)$ compared with cells infected with wild-type Dok-7 ( $n=3$; mean \pm SEM). 
a second that weakly stimulates MuSK and requires phosphorylation of Dok-7 at Y396 and Y406.

\section{Dok-7 tyrosine phosphorylation regulates AChR clustering}

Our experiments indicate that Dok-7 phosphorylation is largely dispensable for at least two aspects of synaptic differentiation: MuSK and AChR- $\beta$ subunit phosphorylation. To determine whether Dok-7 phosphorylation has a role in clustering AChRs, we expressed wild-type or mutant forms of Dok-7 in cultured myotubes and measured AChR clustering by light microscopy. Overexpression of wild-type Dok-7 stimulated an $\sim 50$-fold increase in the number of AChR clusters, which form in the absence of Agrin (Fig. 3). Mutation of either Y396 or Y406 severely impaired the ability of Dok-7 to induce AChR clusters, and mutation of both sites led to a further

A

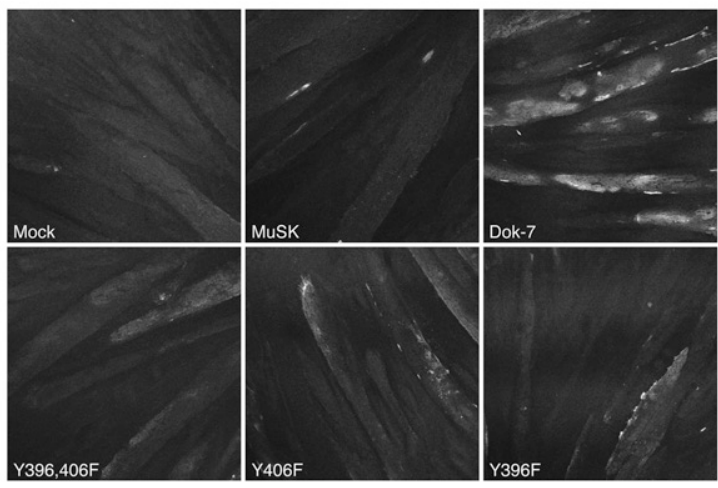

B
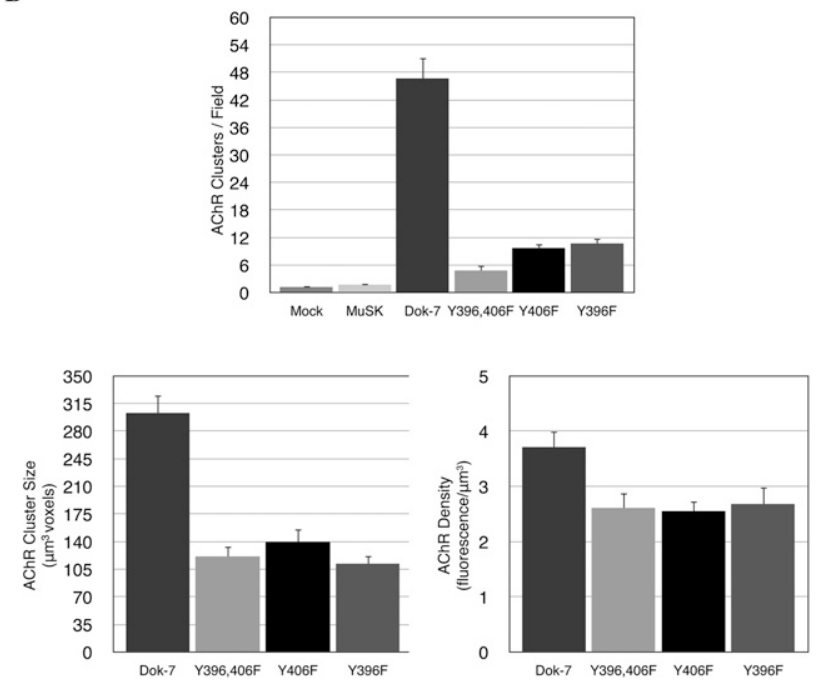

Figure 3. Dok-7 tyrosine phosphorylation plays a critical role in AChR clustering. $(A, B)$ Overexpression of wild-type Dok-7 stimulated AChR clustering ( $\sim 50$-fold), independently of Agrin. Mutation of either Y396 or Y406 impaired AChR clustering, and mutation of both residues reduced AChR clustering still further. Mutation of Y396 or Y406 also led to a reduction in the size of AChR clusters and the density of AChRs within the cluster. Wild-type and mutant proteins were expressed at similar levels (Fig. 2A). decrease (10-fold) in AChR clustering (Fig. 3). Moreover, AChR clusters that formed in myotubes expressing Dok7 mutants were smaller $(\sim 2.5$-fold $)$ than in myotubes expressing wild-type Dok-7 (Fig. 3B). Furthermore, the density of AChRs within these clusters was reduced by $\sim 30 \%$ (Fig. 3B). These data indicate that phosphorylation of Y396 and Y406 plays an important role in AChR clustering.

\section{Phosphorylated Dok-7 peptides purify Crk/Crk-L and Nck1/2 from muscle}

We sought to identify the proteins that are recruited to pY396 and pY406 in Dok-7 and regulate AChR clustering. We synthesized two biotinylated $15 \mathrm{mer}$ phosphopeptides, one centered on pY396 and a second centered on pY406, as well as two control biotinylated peptides, centered on Y396F or Y406F, to isolate binding proteins in lysates from C2 myotubes and 293 cells (Fig. 4A). The phosphopeptides were attached to streptavidinagarose beads, which were incubated with cell lysates from cultured myotubes; the bound proteins were eluted, resolved by SDS-PAGE, and detected by silver staining. A doublet of proteins that migrated at $\sim 37 \mathrm{kDa}$ bound specifically to the pY406 (Fig. 4B) and pY396 peptides (data not shown) but neither control peptide. The proteins contained within this doublet were excised and analyzed by Q-TOF MS, which identified the proteins as Crk-L and Crk-II (Matsuda and Kurata 1996; Feller 2001).

In addition, we probed Western blots of the proteins that bound the phosphopeptide affinity columns with antibodies specific for Crk-I or Crk-II, Crk isoforms that contain one or two SH3 domains, respectively, as well as with antibodies against the related gene, Crk-L. Figure 4C shows that Crk-I, Crk-II, and Crk-L bound to pY396 and pY406 peptides but not to nonphosphorylated control peptides. These data confirm the MS data, showing that Crk-II and Crk-L bind to both phosphopeptides, and demonstrate that Crk-I also binds these sites. Thus, Crk and Crk-L bind directly to Dok-7 phosphorylated at Y396 or Y406.

The sequences surrounding pY396 and pY406 conform to predicted binding sites for the $\mathrm{SH} 2$ domains of Nck1, Nck2, Abl1, and Abl2, as well as Crk and Crk-L (Obenauer et al. 2003). We failed to detect binding of $\mathrm{Abl1} / 2$ to the phosphopeptides by silver staining or Western blotting (Fig. 4B,C). Thus, the accumulation of Abl at synapses is unlikely to be mediated by binding to tyrosine phosphorylated Dok-7 (Finn et al. 2003). Western blotting showed that Nck1/2 bound to the pY406 peptide but not the pY396 peptide (Fig. 4C) in a manner that is mediated by the SH2 domain of Nck (Supplemental Fig. S2). Together, these data demonstrate that phosphorylation of Dok-7 at Y396 and Y406 leads to the recruitment of Crk-I, Crk-II, Crk-L, and Nck1/2, but not Abl.

\section{Agrin stimulates recruitment of Crk-L and Crk to Dok-7}

We next asked whether Crk, Crk-L, and Nck are recruited to Dok-7 following Agrin stimulation. To determine 
A

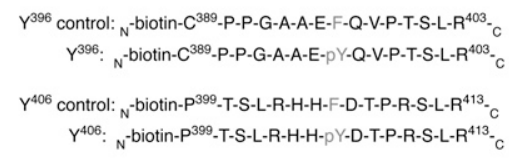

C
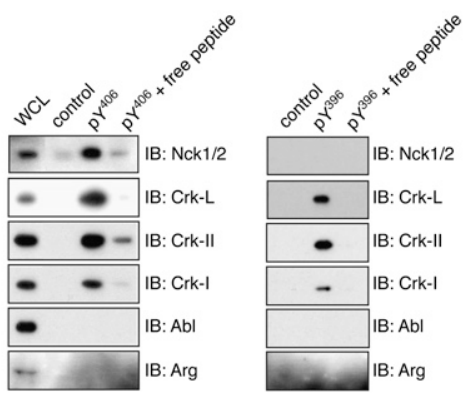

B

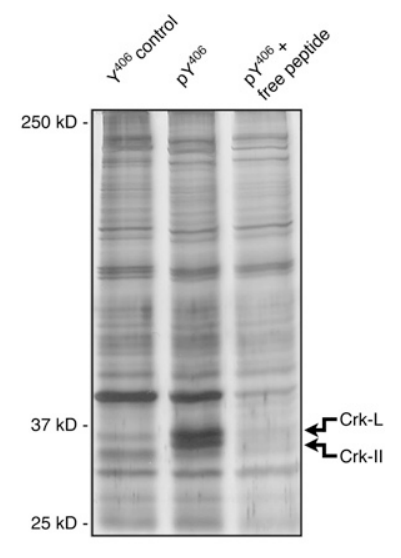

Figure 4. Dok-7 Y396 and Y406 phosphopeptides bind to Crk-L and Crk in myotube lysates. $(A)$ The amino acid sequences of the biotinylated Dok-7 phosphopeptides. (B) The Dok-7 biotin-pY406 phosphopeptide purified an $\sim 37-\mathrm{kDa}$ doublet. The doublet failed to bind the nonphosphorylated biotin-Y406 peptide, and binding to the biotin-pY406 phosphopeptide was inhibited by addition of excess phosphopeptide. The $\sim 37-\mathrm{kDa}$ doublet was identified as Crk-II and Crk-L by Q-TOF MS. $(C)$ Western blots of myotube proteins isolated by binding to pY396 or pY406 phosphopeptides were probed with antibodies to Nck1/2, Crk-I, Crk-II, CrkL, Abl, or Arg. Both phosphopeptides bound Crk-I, CrkII, and Crk-L; in addition, the pY406 phosphopeptide bound Nck1/2. Neither phosphopeptide bound Abl or Arg. See also Supplemental Figure S2. whether Agrin stimulates association of Crk-L and Nck with Dok-7, we stimulated muscle cells with Agrin, isolated Crk-L or Nck by immunoprecipitation, and probed Western blots for Dok-7. Figure 5A shows that Crk-L associated with Dok-7 following Agrin stimulation. In contrast, Nck1/2 failed to associate with Dok-7 under the same conditions (Fig. 5B). To determine whether Crk-I and/or Crk-II associates with Dok-7 in muscle, we treated muscle cells with Agrin, isolated Dok-7 by immunoprecipitation, and probed Western blots with antibodies that recognize both forms of Crk. Figure 5 shows that Crk-II associated with Dok-7 in an Agrin-dependent manner (Fig. 5C). Substantially lower levels of Crk-I coisolated with Dok-7, likely due to the lower expression level of Crk-I in muscle cells (Supplemental Fig. S3). Thus, Agrin stimulation leads to the association of Crk-L and Crk with Dok-7 in skeletal muscle.

\section{Crk and Crk-L are enriched at neuromuscular synapses}

Our data indicate that Crk and Crk-L can associate with tyrosine phosphorylated Dok-7 and suggest that these adapters may have a role in postsynaptic differentiation. To determine whether these adapter proteins are concentrated at neuromuscular synapses, we stained frozen sections of adult and embryonic muscle with antibodies to Crk-L or Crk. Figure 6 shows that both adapter proteins are concentrated at synapses (Fig. 6; Supplemental Fig. S4). Moreover, staining persisted at denervated synapses, showing that labeling is not within nerve terminals (Fig. 6).

\section{Crk and Crk-L play critical roles in neuromuscular synapse formation}

Because Crk and Crk-L bind the same tyrosine phosphorylated sequences in Dok-7 and act redundantly in other cellular contexts (Park and Curran 2008), we considered the possibility that Crk and Crk-L might act redundantly at neuromuscular synapses. We therefore inactivated Crk and $C r k-L$ selectively in skeletal muscle by crossing mice carrying floxed alleles of Crk-L and Crk with myf5 ${ }^{\text {cre }}$ mice, which express Cre recombinase in myoblasts and myotubes, leading to gene inactivation early during muscle development (Tallquist et al. 2000). We failed to recover $\mathrm{Crk}^{f / f}$; Crk- $L^{f / f} ; m y f 5^{\text {cre }}$ mice postnatally, although all other allele combinations were recovered at the expected Mendelian frequency (Supplemental Fig. S5), and their neuromuscular synapses appeared largely normal (data not shown). Because neuromuscular synapses are dispensable during embryonic development but are required for survival at birth, these data raised the possibility that a loss of skeletal muscle Crk and Crk-L led to neonatal lethality. Indeed, at embryonic day 18.5 (E18.5), $1 \mathrm{~d}$ prior to birth, we recovered $\mathrm{Crk}^{f / f}$; Crk $-L^{f / f}$; myf5 $5^{\text {cre }}$ mice at the frequency expected for Mendelian inheritance (data not shown). Moreover, the lungs from newborn $\mathrm{Crk}^{f / f}$; $\mathrm{Crk}_{-} \mathrm{L}^{f / f}$; myf5 $5^{\text {cre }}$ mice failed to expand (Supplemental Fig. S5), indicating that the conditionally mutant mice failed to take a single breath and likely died of respiratory failure.

We analyzed neuromuscular synapses in E18.5 mice by staining whole mounts of muscle with probes that label motor axons, nerve terminals, and AChRs. In wild-type mice, motor axons branch and terminate in a band of synapses adjacent to the main intramuscular nerve in the middle of the muscle. Each muscle fiber is innervated at a single synaptic site, marked by an accumulation of synaptic vesicles in the nerve terminal and a high density of AChRs in the postsynaptic membrane (Burden 1998; Sanes and Lichtman 2001).

Figure 7 shows that mice deficient in skeletal muscle Crk and Crk-L displayed severe defects in presynaptic and postsynaptic differentiation (Fig. 7). First, motor axons were not confined to a narrow endplate band, but were distributed in a broader region of Crk/Crk-L-deficient muscle (Fig. 7A). Second, the number of synapses was reduced by twofold (Fig. 7A,B; Supplemental Fig. S5). The reduction in the number of synapses in Crk/Crk-L-deficient muscle suggested that many muscle fibers lacked innervation. Because it is difficult to distinguish individual 
A

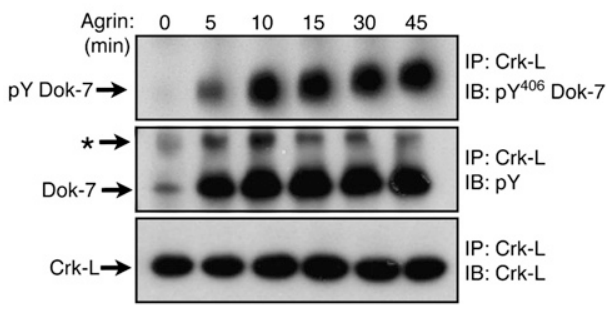

B

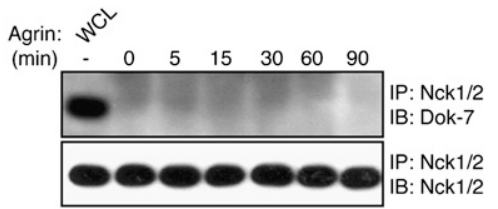

C

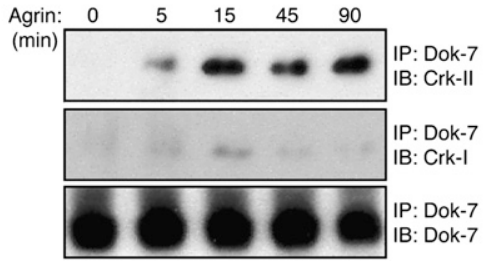

Figure 5. Agrin stimulates recruitment of Crk-L and Crk to a MuSK/Dok-7 signaling complex. (A) Agrin stimulation of C2 myotubes led to the formation of a signaling complex between phosphorylated Dok-7 and Crk-L. Furthermore, two tyrosine phosphorylated proteins, Dok-7, and a $70-\mathrm{kDa}$ protein $\left({ }^{*}\right)$, coimmunoprecipitated with Crk-L. (B) NCK1/2 and Dok7 do not coimmunoprecipitate. $(C)$ Agrin stimulated the formation of a complex containing Dok-7, Crk-I, and Crk-II. See also Supplemental Figure S3.

myofibers in whole mounts, we isolated and stained individual muscle fibers for AChRs. We found that approximately one-half of myofibers in Crk/Crk-L-deficient muscle lacked AChR clusters over their entire length (Fig. 7C), indicating that these myofibers were never innervated or that motor axons retracted from nascent synapses formed earlier during development. This substantial muscle denervation likely contributed to muscle weakness and neonatal lethality. Third, synaptic size was reduced by twofold (Fig. 8A). Fourth, the density of synaptic AChRs was reduced by $25 \%$ (Fig. 8B). Synaptic AChRs were tyrosine phosphorylated (Supplemental Fig. S5), consistent with the minor role of Dok-7 phosphorylation in regulating AChR phosphorylation in vitro (Fig. 2). Thus, Crk/Crk-L mutant synapses contain approximately threefold fewer AChRs than normal synapses. Because the safety factor for synaptic transmission at adult mouse neuromuscular synapses is 2.5 to 4 (Harris and Ribchester 1976; Wood and Slater 2001), and less at neonatal synapses (Wood and Slater 2001), this reduction in AChR number may compromise the reliability of synaptic transmission and contribute to the neonatal lethality of mice deficient in muscle Crk and Crk-L. Finally, motor axons often failed to terminate at synapses and instead continued to grow beyond AChR clusters (Figs. 8C; Supplemental Fig. S5), resembling terminal sprouts found in adult muscle after inhibiting synaptic transmission or following reinnervation (Brown and Ironton 1977; Betz et al. 1980; Son and Thompson 1995). Thus, muscle-derived Crk/Crk-L fulfills a critical role in neuromuscular synapse formation, as these adaptor proteins are essential to cluster AChRs at synapses, regulate synaptic size, and control motor axon growth.

\section{Discussion}

Agrin stimulates tyrosine phosphorylation of MuSK, leading to the recruitment and tyrosine phosphorylation of Dok-7 (Okada et al. 2006). The steps following Dok-7 tyrosine phosphorylation are poorly understood. Here, we show that Agrin stimulates phosphorylation of two tyrosine residues in the C-terminal domain of Dok-7, which leads to the recruitment of two adaptor proteins: Crk and Crk-L. Furthermore, we demonstrate that Dok-7 tyrosine phosphorylation regulates the number and size of AChR clusters that form in cultured muscle cells, and provide genetic evidence supporting a redundant role for Crk and Crk-L in the regulation of both presynaptic and postsynaptic differentiation in vivo. These data indicate that Crk and Crk-L are key components in the Agrin/MuSK signaling pathway that function downstream from Dok-7.

A

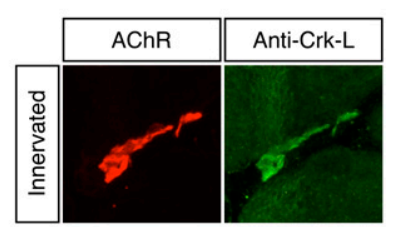

B
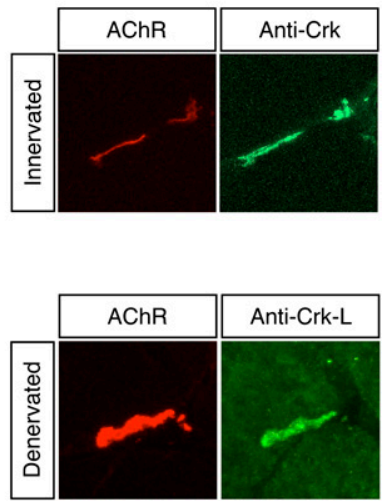

Figure 6. Crk-L and Crk are enriched at neuromuscular synapses. Cross-sections of innervated and denervated adult rat gastrocnemius muscles were stained with Alexa-594 $\alpha$-BGT, which labels synapses (red), and antibodies to Crk-L or Crk-I/II (green). Crk-L $(A)$ and Crk-I/II $(B)$ staining were enriched at synaptic sites. $(C)$ Crk-L was concentrated in the postsynaptic membrane, since staining remained at denervated synaptic sites. 
A

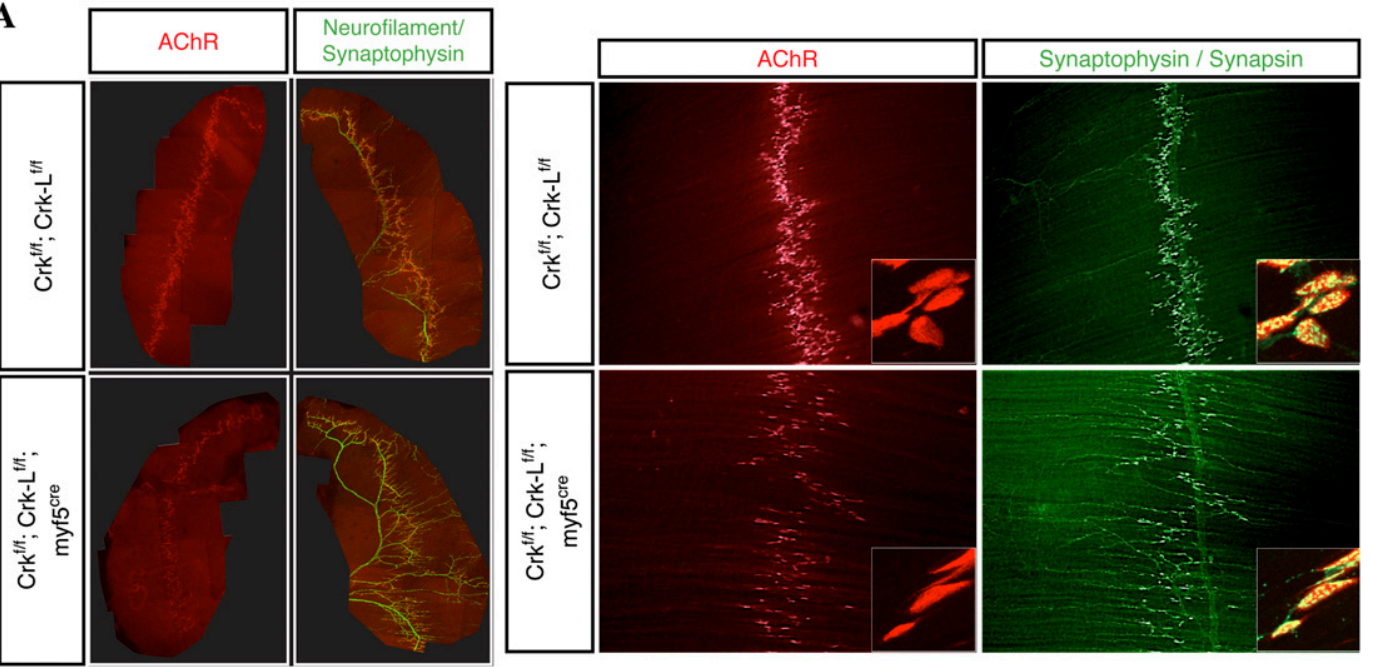

B

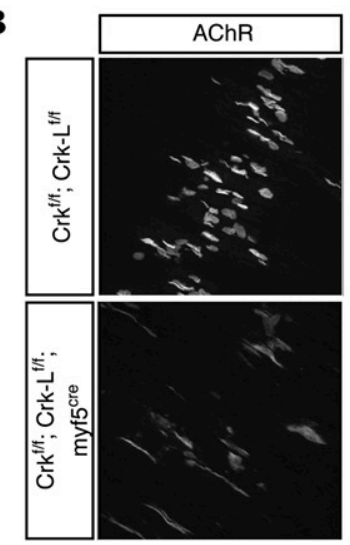

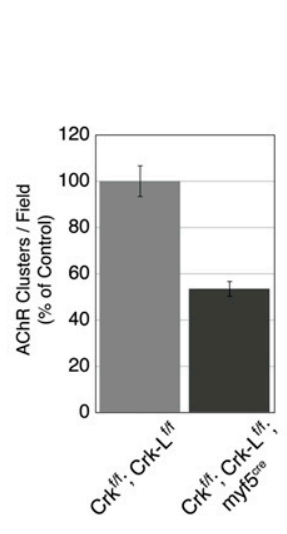

$\mathbf{C}$

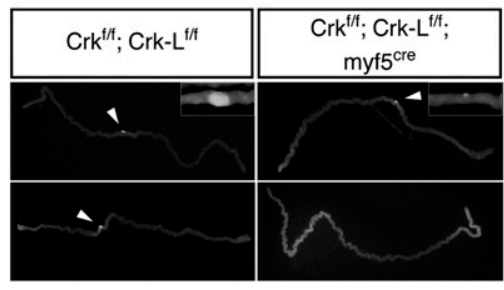

No Receptor

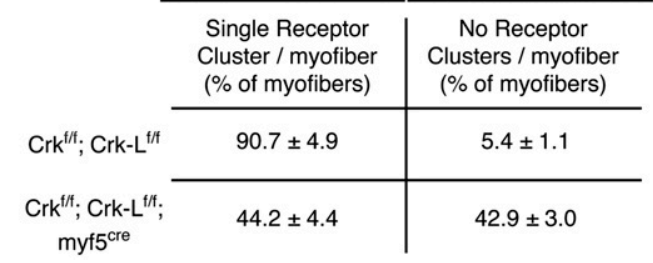

Figure 7. Crk and Crk-L play critical roles in neuromuscular synapse formation. $(A, B)$ Muscles from E18.5 mice deficient in muscle Crk/Crk-L contained fewer synapses than wild-type mice, and these synapses were broadly distributed in the muscle (Supplemental Fig. S5). (C) Indeed, approximately one-half of myofibers in Crk/Crk-L-deficient muscle lacked innervation, as AChR clusters were absent over the entire myofiber length. For $A$ and $B, n=3$, and error bars indicate standard errors of the means. For $C, n=2$. See also Supplemental Figure S5.

Dok-7 is essential for Agrin to stimulate MuSK tyrosine phosphorylation. This unusual feature of an adapter protein raised the possibility that Dok-7 functions at synapses solely by stimulating MuSK phosphorylation rather than by recruiting additional signaling components that act downstream from MuSK. Multiple lines of evidence support the notion that Dok-7 is required for MuSK phosphorylation. First, mutations in MuSK, which prevent Dok-7 recruitment, block Agrin-stimulated MuSK phosphorylation (Zhou et al. 1999; Herbst and Burden 2000). Second, Agrin fails to stimulate MuSK phosphorylation in myotubes deficient in Dok-7 (Inoue et al. 2009). Third, Dok-7 overexpression stimulates MuSK phosphorylation in the absence of Agrin (Okada et al. 2006). Here, we show that Dok-7 functions both to amplify MuSK phosphorylation and recruit additional components that promote postsynaptic differentiation. As such, these findings help clarify how Dok-7 mutations, which result in loss of the C-terminal domain, cause CMS (Beeson et al. 2006).

Dok-7 appears to act by two distinct pathways to stimulate synaptic differentiation. The $\mathrm{N}$-terminal region of Dok-7, containing the PH and PTB domains, stimulates MuSK phosphorylation (Inoue et al. 2009; Bergamin et al. 2010) and minimal features of postsynaptic differentiation by a pathway that acts independently of Crk/ Crk-L recruitment to Dok-7 (Beeson et al. 2006; Muller et al. 2007; Selcen et al. 2008). In contrast, the C-terminal region of Dok-7 has a modest role in stimulating MuSK but is essential for complete synaptic differentiation. As such, humans with mutations that truncate the C-terminal domain of Dok-7 form primitive synapses and develop CMS. Understanding how these two Dok-7 pathways regulate synaptic differentiation will hopefully lead to insights for enhancing synaptic differentiation and improving neuromuscular function in patients with Dok-7 CMS.

How Dok-7 recruitment stimulates MuSK phosphorylation is not completely understood. Phosphorylation of Dok-7 at Y396 and Y406 has a modest role in stimulating MuSK phosphorylation, and this effect may be mediated by a kinase, possibly Abll/2, that is recruited to Crk/ Crk-L. Nonetheless, the major stimulatory effect occurs 

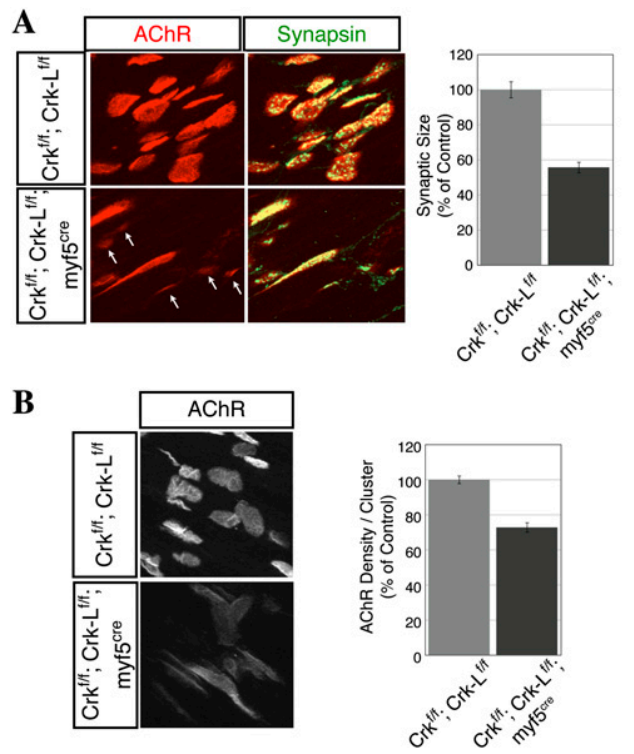

C

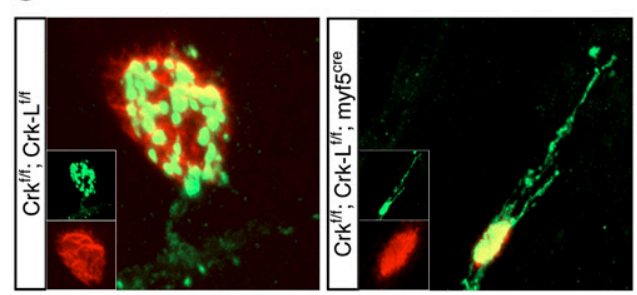

Figure 8. Neuromuscular synapses are aberrant in Crk- and Crk-L-deficient muscle. In Crk/Crk-L-deficient muscle, synapses were unusually small (arrows in $A$ ), and the density of synaptic AChRs was reduced $(B) .(C)$ In Crk/Crk-L-deficient muscle, motor axons contacted AChR clusters but failed to stop growing. For $A$ and $B, n=3$, and error bars indicate standard errors of the means. For $C, n=2$.

independently of Dok-7 tyrosine phosphorylation (Inoue et al. 2009; Bergamin et al. 2010). Our biochemical and structural studies demonstrate that Dok-7 forms a homodimer mediated by interactions between the PH and PTB domains in adjacent molecules, and suggest that Dok7 increases MuSK phosphorylation by binding MuSK and stabilizing MuSK dimers (Inoue et al. 2009; Bergamin et al. 2010). In this model, a low level of MuSK juxtamembrane phosphorylation would stimulate recruitment of Dok-7, promote MuSK dimers and MuSK activation loop phosphorylation, increase MuSK kinase activity, and lead to juxtamembrane phosphorylation on additional MuSK molecules, thereby propagating Dok-7 recruitment and amplifying MuSK activation. This pathway, however, is likely to be inefficient or restrained in vivo, as MuSK is poorly phosphorylated in the absence of Agrin (Gautam et al. 1996; Glass et al. 1996). Maintaining Dok-7 expression at low levels may be sufficient to minimize this Agrin-independent activity; alternatively, negative regulators may hinder Dok-7 recruitment.

Synapses fail to form in mice that are homozygous for null mutations in MuSK or Dok-7, leading to neonatal lethality. In contrast, hypomorphic alleles of Dok-7, which truncate Dok-7 and lead to a loss of Y396 and Y406, retain sufficient activity to mediate the formation of synapses. These synapses are nonetheless structurally and functionally defective; in particular, the synapses are small and motor axons fail to reliably terminate, leading to muscle weakness and fatigue in adults, the hallmark features of CMS (Beeson et al. 2006; Muller et al. 2007; Selcen et al. 2008). Thus, these truncated forms of Dok-7, which cannot recruit Crk/Crk-L, mediate the formation of simplified and poorly functional synapses. Importantly, these defective synapses resemble the aberrant synapses found in mice deficient in muscle-derived Crk/Crk-L.

Identification of the role of Crk and Crk-L in the Agrin/ MuSK pathway offers some insights into the mechanism responsible for neuromuscular synapse formation, as Crk and Crk-L have been studied extensively in a variety of biological processes. SH3 domains, present in noncatalytic adaptor proteins such as Crk/Crk-L, serve as recognition sites for a large number of proteins with prolinerich regions (Ren et al. 1993; Pawson and Nash 2003), including guanine nucleotide exchange factors (GEFs) and actin remodeling factors (Li et al. 2001; Akakura et al. 2005; Antoku and Mayer 2009). Indeed, C3G (a GEF protein) and Rap1 (a small GTPase) have been proposed to act downstream from Crk and Crk-L in the Reelin pathway (Ballif et al. 2004; Voss et al. 2008). On the other hand, several lines of evidence indicate that Rho family members and actin remodeling factors have a key role in Agrin-stimulated AChR clustering. First, Agrin stimulates nucleotide exchange in Rac and Rho (Weston et al. 2000, 2003); second, inhibiting Rac or Rho interferes with Agrin-stimulated AChR clustering (Weston et al. 2003); third, Agrin stimulates phosphorylation of Pak, which is activated by Rac and regulates actin dynamics (Luo et al. 2002); fourth, actin polymerizes at AChR clusters in cultured cells and is concentrated at neuromuscular synapses (Dai et al. 2000); and fifth, inhibiting actin assembly with latrunculin interferes with Agrin-stimulated AChR clustering (Dai et al. 2000; Weston et al. 2000). Together, these data raise the possibility that Crk/Crk-L, recruited to tyrosine phosphorylated Dok-7, forms complexes with Rac/Rho GEFs, such as Dock180-related GEFs or C3G (Feller 2001; Miyamoto and Yamauchi 2010), and links MuSK activation to a change in actin dynamics and a redistribution of postsynaptic proteins, including AChRs.

The SH3 domains in Crk/Crk-L can also bind Abll and Abl2 (Feller et al. 1994), nonreceptor tyrosine kinases that have been implicated in clustering AChRs (Finn et al. 2003). Because Abl also regulates actin dynamics (Lanier and Gertler 2000; Zandy et al. 2007), Crk/Crk-L may serve as an integrator for multiple pathways, including a Rac/Rho/Pak pathway and an Abl/Ena/Vasp pathway, to regulate cytoskeletal reorganization and AChR clustering following Agrin stimulation.

Our experiments demonstrate a second instance in which Crk and Crk-L function in a redundant manner downstream from a Lrp. Reelin, a ligand that binds two Lrps, Lrp8 and VLDLR, stimulates tyrosine phosphorylation of Disabled-1 (Dab1), a PTB domain-containing adapter protein that recruits Crk and Crk-L to control 
neuronal positioning in the developing brain (Rice and Curran 2001; Ayala et al. 2007). These results raise the possibility that a tyrosine kinase signaling module, composed of a PTB adapter protein such as Dok-7 or Dab1, together with Crk/Crk-L, may function in a similar manner downstream from other Lrps. It will be particularly interesting to learn whether Crk and Crk-L function downstream from Lrp1, which, like Lrp4, is expressed in the nervous system and concentrated in postsynaptic densities (May et al. 2004).

Very few genes are known to act downstream from MuSK and regulate synapse formation in vivo. Rapsyn, a peripheral membrane protein associated with AChRs, is critical for presynaptic and postsynaptic differentiation, as shown by genetic studies in mice (Gautam et al. 1995). Tid1 and Dishevelled, two noncatalytic adapter proteins, bind MuSK and function in a poorly understood manner downstream from MuSK in cultured myotubes (Luo et al. 2002; Linnoila et al. 2008). Genetic analysis of mice lacking these genes, however, has not been described. Likewise, although Rac, Rho, Abl, Arg, Pak, Cortactin, and Actin have been implicated to regulate AChR clustering in cultured myotubes (Wu et al. 2010), there is little genetic evidence demonstrating a role for these proteins in synapse formation in vivo. A better understanding of synapse formation and maintenance will require knowledge of how these genes function at synapses in vivo, and how they act in concert with Crk/Crk-L.

\section{Materials and methods}

\section{DNA constructs}

Murine Dok-7 (GenBank: BAE96740) cDNA was isolated by PCR from C2 muscle cell cDNA and subcloned into pIRES2-EGFP for expression in HEK 293 cells or into pBabe-puro for expression in murine $\mathrm{C} 2$ muscle cells. Tyrosine residues in Dok-7 were mutated to phenylalanine by site-directed mutagenesis. Myc-MuSK-GFP was expressed in 293 or C2 cells by infecting with pBabe-mycMuSK-GFP, as described previously (Herbst and Burden 2000). The structure of all constructs was confirmed by DNA sequencing. Ecotropic retrovirus particles were generated by transfecting Phoenix cells with pBabe-Dok-7 constructs (Kinsella and Nolan 1996). C2 myoblasts were infected, selected for puromycin resistance, and allowed to differentiate into myotubes.

\section{Phosphopeptide pull-down assay}

Dok-7 Y396 and Y406 biotinylated phosphopeptides (Invitrogen) were immobilized to streptavidin agarose and incubated with a detergent-soluble lysate (5 mg of protein) from C2 myotubes. Phosphopeptide-specific binding proteins were eluted by the addition of excess free biotin and resolved by SDS-PAGE. Following silver staining, proteins were extracted from gel slices and analyzed by MS or Western blotting. Antibodies to Dok-7 pY396 or pY406 were cross-absorbed with the opposite phosphopeptide prior to Western blotting.

\section{AChR clustering assay}

Dok-7-infected C2 myotubes were stained with Alexa-594$\alpha$-bungarotoxin $(\alpha-B G T)$ and imaged using a Zeiss LSM 510 confocal microscope. The number and size of AChR clusters, as well as the density of AChRs within AChR clusters, were determined by imaging at least 10 fields in triplicate samples using Volocity 3D imaging software (Perkin Elmer), as described previously (Friese et al. 2007).

\section{Antibodies}

Rabbit and chicken antibodies to the C-terminal region of Dok-7 have been described previously (Kim et al. 2008). We also used antibodies to Dok-7 Y396 or Y406 phosphopeptides (sc-68691 and sc-68692 from Santa Cruz Biotechnology). MuSK was immunoprecipitated using antibodies to the $\mathrm{C}$ terminus of MuSK (Herbst and Burden 2000) and was detected by probing Western blots with antibodies to MuSK (AF3904 from R\&D Systems). A monoclonal antibody to the AChR subunit (mab124) was the kind gift of Dr. J. Lindstrom (University of Pennsylvania). Antibodies to Nck1/2 were the kind gift of Dr. T. Pawson (Samuel Lunenfeld Institute) or were purchased from BD Biosciences. We also used antibodies to Abl (mab 8E9 from BD Biosciences), Arg (C-20 from Santa Cruz Biotechnology), phosphotyrosine (4G10 from Millipore), Crk-I/-II (mab 22 from BD Biosciences), Crk-II (C18 from Santa Cruz Biotechnology), Crk-L (C20 from Santa Cruz Biotechnology), and Crk-L (GeneTex). We established the specificity of the antibodies to Crk (mab 22) and Crk-L (C20) by probing Western blots of lysates from Crk or Crk- $L$ mutant embryos: The antibodies to Crk react specifically with Crk and do not cross-react with Crk-L; likewise, antibodies to Crk-L react selectively with Crk-L (Supplemental Fig. S6). Infrared-conjugated secondary antibodies were from LI-COR.

\section{Sample preparation and LC-MS/MS analysis of Dok-7,} Crk-L, and Crk-II

Gel bands containing Dok-7, Crk-L, and Crk-II were excised from SDS-polyacrylamide gels and digested using MS-grade trypsin (Promega). For protein identification, the digests were analyzed by LC/ESI-MS/MS, using a nanoAquity UPLC coupled directly to a Q-TOF Premier MS (Waters). The proteins were identified using MASCOT (MatrixScience) to search the NCBInr mouserat protein sequence database. To identify phosphorylation sites in Dok-7, phosphopeptides were enriched from tryptic digests by titanium dioxide chromatography, and were analyzed using nanoflow LC/ESI-MS/MS with a NanoLC-2D system (Eksigent) coupled directly to an LTQ-Orbitrap mass spectrometer (Thermo Fisher Scientific). Phosphopeptides were identified using MASCOT in normal and then error-tolerant mode. The identities of phosphopeptides and sites of tyrosine phosphorylation were verified by manual inspection.

\section{Animals}

Mice were housed and maintained according to International Animal Care and Use Committee guidelines. Mice carrying floxed alleles of Crk and Crk- $L$ have been described previously (Park et al. 2006; Park and Curran 2008). Mice that carry a myf5 $5^{\text {cre }}$ allele, which confers Cre recombinase expression in myoblasts and myotubes, have been described previously (Tallquist et al. 2000). Whole mounts of diaphragm muscles from E18.5 mice were stained with antibodies to neurofilament (AB 1981 from Millipore), Synaptophysin (clone Z66, Invitrogen), and/or synapsin (106 002, SYnaptic SYstems) to label axons and nerve terminals. Images were acquired with a Zeiss LSM 510 confocal microscope; the size and number of AChR clusters, as well as the density of AChRs within AChR clusters, were determined using Volocity 3D imaging software, as described previously (Jaworski and Burden 2006). Three-dimensional movies were generated 
using software from Imaris (Bitplane Scientific Software). Myofibers were dissociated from E18.5 muscle and stained for AChRs as described previously (Shefer and Yablonka-Reuveni 2005; Kim and Burden 2008).

\section{Acknowledgments}

We thank Tony Pawson for antibodies to Nck1/2 and for GSTNck fusion proteins, Jon Lindstrom for mab124 to the AChR, and Stevan Hubbard for helpful discussions and advice. We are grateful to Sabrina Suckiel for excellent technical assistance. This work was supported with funds from the National Institutes of Health (AR055027 to S.J.B., and P30 NS050276 and S10 RR017990 to T.A.N.), and by a Pennsylvania Department of Health Cure Formulary grant (SAP no. 410047678 to T.C.).

\section{References}

Akakura S, Kar B, Singh S, Cho L, Tibrewal N, SanokawaAkakura R, Reichman C, Ravichandran KS, Birge RB. 2005. C-terminal SH3 domain of CrkII regulates the assembly and function of the DOCK180/ELMO Rac-GEF. I Cell Physiol 204: 344-351.

Antoku S, Mayer BJ. 2009. Distinct roles for Crk adaptor isoforms in actin reorganization induced by extracellular signals. J Cell Sci 122: 4228-4238.

Ayala R, Shu T, Tsai LH. 2007. Trekking across the brain: The journey of neuronal migration. Cell 128: 29-43.

Ballif BA, Arnaud L, Arthur WT, Guris D, Imamoto A, Cooper JA. 2004. Activation of a Dab1/CrkL/C3G/Rap1 pathway in Reelin-stimulated neurons. Curr Biol 14: 606-610.

Beeson D, Higuchi O, Palace J, Cossins J, Spearman H, Maxwell S, Newsom-Davis J, Burke G, Fawcett P, Motomura M, et al. 2006. Dok-7 mutations underlie a neuromuscular junction synaptopathy. Science 313: 1975-1978.

Bergamin E, Hallock PT, Burden SJ, Hubbard SR. 2010. The cytoplasmic adaptor protein Dok7 activates the receptor tyrosine kinase MuSK via dimerization. Mol Cell 39: 100109.

Betz WJ, Caldwell JH, Ribchester RR. 1980. Sprouting of active nerve terminals in partially inactive muscles of the rat. J Physiol 303: 281-297.

Brown MC, Ironton R. 1977. Motor neurone sprouting induced by prolonged tetrodotoxin block of nerve action potentials. Nature 265: 459-461.

Burden SJ. 1998. The formation of neuromuscular synapses. Genes Dev 12: 133-148.

Dai Z, Luo X, Xie H, Peng HB. 2000. The actin-driven movement and formation of acetylcholine receptor clusters. J Cell Biol 150: 1321-1334.

Feller SM. 2001. Crk family adaptors-signalling complex formation and biological roles. Oncogene 20: 6348-6371.

Feller SM, Knudsen B, Hanafusa H. 1994. c-Abl kinase regulates the protein binding activity of c-Crk. EMBO J 13: 2341-2351.

Finn AJ, Feng G, Pendergast AM. 2003. Postsynaptic requirement for Abl kinases in assembly of the neuromuscular junction. Nat Neurosci 6: 717-723.

Friese MB, Blagden CS, Burden SJ. 2007. Synaptic differentiation is defective in mice lacking acetylcholine receptor $\beta$-subunit tyrosine phosphorylation. Development 134: 4167-4176.

Gautam M, Noakes PG, Mudd J, Nichol M, Chu GC, Sanes JR, Merlie JP. 1995. Failure of postsynaptic specialization to develop at neuromuscular junctions of rapsyn-deficient mice. Nature 377: 232-236.

Gautam M, Noakes PG, Moscoso L, Rupp F, Scheller RH, Merlie JP, Sanes JR. 1996. Defective neuromuscular synaptogenesis in agrin-deficient mutant mice. Cell 85: 525-535.
Glass DJ, Bowen DC, Stitt TN, Radziejewski C, Bruno J, Ryan TE, Gies DR, Shah S, Mattsson K, Burden SI, et al. 1996. Agrin acts via a MuSK receptor complex. Cell 85: 513-523.

Hamuro J, Higuchi O, Okada K, Ueno M, Iemura S, Natsume T, Spearman H, Beeson D, Yamanashi Y. 2008. Mutations causing DOK7 congenital myasthenia ablate functional motifs in Dok-7. J Biol Chem 283: 5518-5524.

Harris JB, Ribchester RR. 1976. Neuromuscular transmission in the isolated diaphragm of the dystrophic mouse (129 ReJ/ dy.dy). J Physiol 263: 118P-119P.

Herbst R, Burden SJ. 2000. The juxtamembrane region of MuSK has a critical role in agrin-mediated signaling. EMBO $J$ 19: 67-77.

Inoue A, Setoguchi K, Matsubara Y, Okada K, Sato N, Iwakura Y, Higuchi O, Yamanashi Y. 2009. Dok-7 activates the muscle receptor kinase MuSK and shapes synapse formation. Sci Signal 2: ra7. doi: 10.1126/scisignal.2000113.

Jaworski A, Burden SJ. 2006. Neuromuscular synapse formation in mice lacking motor neuron- and skeletal muscle-derived Neuregulin-1. I Neurosci 26: 655-661.

Jennings CG, Dyer SM, Burden SJ. 1993. Muscle-specific trkrelated receptor with a kringle domain defines a distinct class of receptor tyrosine kinases. Proc Natl Acad Sci 90: 2895-2899.

Kim N, Burden SJ. 2008. MuSK controls where motor axons grow and form synapses. Nat Neurosci 11: 19-27.

Kim N, Stiegler AL, Cameron TO, Hallock PT, Gomez AM, Huang JH, Hubbard SR, Dustin ML, Burden SJ. 2008. Lrp4 is a receptor for Agrin and forms a complex with MuSK. Cell 135: 334-342.

Kinsella TM, Nolan GP. 1996. Episomal vectors rapidly and stably produce high-titer recombinant retrovirus. Hum Gene Ther 7: 1405-1413.

Lanier LM, Gertler FB. 2000. From Abl to actin: Abl tyrosine kinase and associated proteins in growth cone motility. Curr Opin Neurobiol 10: 80-87.

Li W, Fan J, Woodley DT. 2001. Nck/Dock: An adapter between cell surface receptors and the actin cytoskeleton. Oncogene 20: 6403-6417.

Linnoila J, Wang Y, Yao Y, Wang ZZ. 2008. A mammalian homolog of Drosophila tumorous imaginal discs, Tid1, mediates agrin signaling at the neuromuscular junction. Neuron 60: 625-641.

Luo ZG, Wang Q, Zhou JZ, Wang J, Luo Z, Liu M, He X, Wynshaw-Boris A, Xiong WC, Lu B, et al. 2002. Regulation of AChR clustering by Dishevelled interacting with MuSK and PAK1. Neuron 35: 489-505.

Matsuda M, Kurata T. 1996. Emerging components of the Crk oncogene product: The first identified adaptor protein. Cell Signal 8: 335-340.

May P, Rohlmann A, Bock HH, Zurhove K, Marth JD, Schomburg ED, Noebels JL, Beffert U, Sweatt JD, Weeber EJ, et al. 2004. Neuronal LRP1 functionally associates with postsynaptic proteins and is required for normal motor function in mice. Mol Cell Biol 24: 8872-8883.

Miyamoto Y, Yamauchi J. 2010. Cellular signaling of Dock family proteins in neural function. Cell Signal 22: 175182.

Muller JS, Herczegfalvi A, Vilchez JJ, Colomer J, Bachinski LL, Mihaylova V, Santos M, Schara U, Deschauer M, Shevell M, et al. 2007. Phenotypical spectrum of DOK7 mutations in congenital myasthenic syndromes. Brain 130: 14971506.

Obenauer JC, Cantley LC, Yaffe MB. 2003. Scansite 2.0: Proteome-wide prediction of cell signaling interactions using short sequence motifs. Nucleic Acids Res 31: 3635-3641. 
Okada K, Inoue A, Okada M, Murata Y, Kakuta S, Jigami T, Kubo S, Shiraishi H, Eguchi K, Motomura M, et al. 2006. The muscle protein Dok-7 is essential for neuromuscular synaptogenesis. Science 312: 1802-1805.

Park TJ, Curran T. 2008. Crk and Crk-like play essential overlapping roles downstream of disabled-1 in the Reelin pathway. J Neurosci 28: 13551-13562.

Park TJ, Boyd K, Curran T. 2006. Cardiovascular and craniofacial defects in Crk-null mice. Mol Cell Biol 26: 6272-6282.

Pawson T, Nash P. 2003. Assembly of cell regulatory systems through protein interaction domains. Science 300: 445-452.

Ren R, Mayer BJ, Cicchetti P, Baltimore D. 1993. Identification of a ten-amino acid proline-rich $\mathrm{SH} 3$ binding site. Science 259: 1157-1161.

Rice DS, Curran T. 2001. Role of the reelin signaling pathway in central nervous system development. Annu Rev Neurosci 24: 1005-1039.

Sanes JR, Lichtman JW. 2001. Induction, assembly, maturation and maintenance of a postsynaptic apparatus. Nat Rev Neurosci 2: 791-805.

Selcen D, Milone M, Shen XM, Harper CM, Stans AA, Wieben ED, Engel AG. 2008. Dok-7 myasthenia: Phenotypic and molecular genetic studies in 16 patients. Ann Neurol 64: 71-87.

Shefer G, Yablonka-Reuveni Z. 2005. Isolation and culture of skeletal muscle myofibers as a means to analyze satellite cells. Methods Mol Biol 290: 281-304.

Son YJ, Thompson WJ. 1995. Nerve sprouting in muscle is induced and guided by processes extended by Schwann cells. Neuron 14: 133-141.

Tallquist MD, Weismann KE, Hellstrom M, Soriano P. 2000. Early myotome specification regulates PDGFA expression and axial skeleton development. Development 127: 50595070.

Valenzuela DM, Stitt TN, DiStefano PS, Rojas E, Mattsson K, Compton DL, Nunez L, Park JS, Stark JL, Gies DR, et al. 1995. Receptor tyrosine kinase specific for the skeletal muscle lineage: Expression in embryonic muscle, at the neuromuscular junction, and after injury. Neuron 15: 573584.

Vogt J, Morgan NV, Marton T, Maxwell S, Harrison BJ, Beeson D, Maher ER. 2009. Germline mutation in DOK7 associated with fetal akinesia deformation sequence. I Med Genet 46: 338-340.

Voss AK, Britto JM, Dixon MP, Sheikh BN, Collin C, Tan SS, Thomas T. 2008. C3G regulates cortical neuron migration, preplate splitting and radial glial cell attachment. Development 135: 2139-2149.

Weatherbee SD, Anderson KV, Niswander LA. 2006. LDLreceptor-related protein 4 is crucial for formation of the neuromuscular junction. Development 133: 4993-5000.

Weston C, Yee B, Hod E, Prives J. 2000. Agrin-induced acetylcholine receptor clustering is mediated by the small guanosine triphosphatases Rac and Cdc42. J Cell Biol 150: 205-212.

Weston C, Gordon C, Teressa G, Hod E, Ren XD, Prives J. 2003. Cooperative regulation by $\mathrm{Rac}$ and Rho of agrin-induced acetylcholine receptor clustering in muscle cells. I Biol Chem 278: 6450-6455.

Wood SJ, Slater CR. 2001. Safety factor at the neuromuscular junction. Prog Neurobiol 64: 393-429.

Wu H, Xiong WC, Mei L. 2010. To build a synapse: Signaling pathways in neuromuscular junction assembly. Development 137: 1017-1033.

Zandy NL, Playford M, Pendergast AM. 2007. Abl tyrosine kinases regulate cell-cell adhesion through Rho GTPases. Proc Natl Acad Sci 104: 17686-17691.
Zhang B, Luo S, Wang Q, Suzuki T, Xiong WC, Mei L. 2008. LRP4 serves as a coreceptor of agrin. Neuron 60: 285-297.

Zhou H, Glass DI, Yancopoulos GD, Sanes JR. 1999. Distinct domains of MuSK mediate its abilities to induce and to associate with postsynaptic specializations. J Cell Biol 146: 1133-1146. 


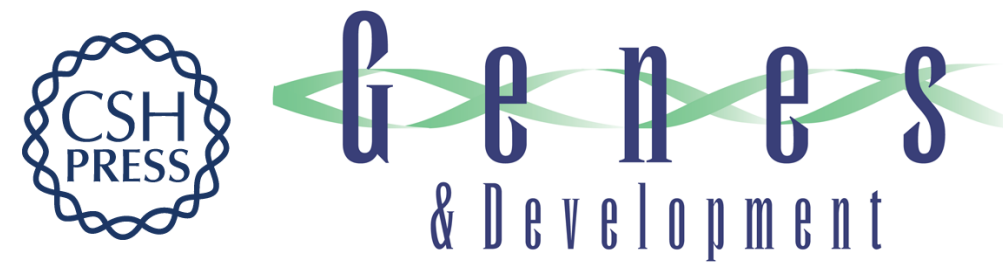

\section{Dok-7 regulates neuromuscular synapse formation by recruiting Crk and Crk-L}

Peter T. Hallock, Chong-Feng Xu, Tae-Ju Park, et al.

Genes Dev. 2010, 24:

Access the most recent version at doi:10.1101/gad.1977710

Supplemental http://genesdev.cshlp.org/content/suppl/2010/10/21/24.21.2451.DC1
Material

References This article cites 57 articles, 25 of which can be accessed free at:

http://genesdev.cshlp.org/content/24/21/2451.full.html\#ref-list-1

License

Email Alerting

Receive free email alerts when new articles cite this article - sign up in the box at the top

Service

right corner of the article or click here.

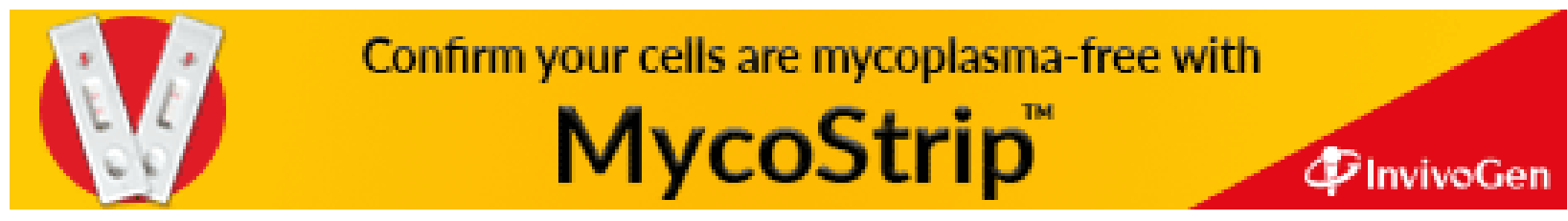

